\title{
Design of Erosion/Abrasion Studies - Insights and Rational Concepts
}

\author{
Annette Wiegand Thomas Attin \\ Clinic for Preventive Dentistry, Periodontology and Cariology, Centre for Dental and Oral Medicine and \\ Craniomaxillofacial Surgery, University of Zurich, Zurich, Switzerland
}

\section{Key Words}

Abrasion · Acid · Brushing • Erosion • In situ studies •

In vitro studies $\cdot$ Toothbrush $\cdot$ Toothpaste

\begin{abstract}
In vitro and in situ studies modelling the wear of dental hard tissues due to erosion and abrasion are characterised by a high variation in study designs and experimental parameters. Based on a summary of the existing protocols, the present review aimed to describe and discuss the parameters which must be carefully considered in erosion-abrasion research, especially when it is intended to simulate clinical conditions. Experimental characteristics and parameters were retrieved from a total of 42 in vitro and 20 in situ studies. The key experimental characteristics included parameters of erosion (duration and $\mathrm{pH}$ ) and abrasion (duration, kinds of toothbrush and toothpaste, brushing force, and time point) as well as co-factors (e.g. dental hard tissue). The majority of studies used models with alternating erosion/abrasion treatments intended to simulate clinical conditions, while other studies exaggerated clinical conditions intentionally, often using only a single erosion/abrasion treatment. Both in vitro and in situ models shared a high level of standardisation, but several studies showed a trend to severe erosion (e.g. $>5 \mathrm{~min} / \mathrm{cycle}$ ) or extensive brushing (e.g. $>100$ brushing
\end{abstract}

strokes/cycle) at a high frequency and repetition rate. Thus, studies often tend to produce a higher amount of wear than in the clinical situation, especially as modifying biological factors (e.g. the dilution of the erosive solution by saliva and the protective effect of the pellicle) cannot be simulated adequately. With respect to the existing models, it seems advisable to diminish duration and frequency of erosion and abrasion to more realistic clinical conditions when the everyday situation is to be simulated. Experimental parameters must be chosen with care to ensure that the problem is investigated in an appropriate mode at standardised conditions and with adequate measuring systems to allow prediction of clinical outcomes.

Copyright $\odot 2011$ S. Karger AG, Basel

In recent years, extensive research has been undertaken in order to gain a better insight into and understanding of the processes involved in the development of erosive tooth wear. Erosion, abrasion and attrition rarely act alone but interact with each other, and abrasion of erosively altered dental hard tissues is considered as the most important interaction. Thus, numerous studies have investigated either the effects of tooth brushing on eroded dental hard tissues per se or the efficacy of different preventive agents under combined erosive/abrasive conditions. In

\section{KARGER}

Fax +4161306 1234

E-Mail karger@karger.ch

www.karger.com
(C) 2011 S. Karger AG, Basel

0008-6568/11/0457-0053\$38.00/0

Accessible online at:

www.karger.com/cre
PD Dr. A. Wiegand

Clinic for Preventive Dentistry, Periodontology and Cariology

Centre for Dental and Oral Medicine and Craniomaxillofacial Surgery

University of Zurich, Plattenstrasse 11, CH-8032 Zürich (Switzerland)

Tel.+41 446343 2701, E-Mail annette.wiegand@zzm.uzh.ch 
Table 1. Recommendations for adjustment of variables to simulate intra-oral real-life conditions as closely as possible in in vitro and in situ models testing erosion/abrasion of dental hard tissue

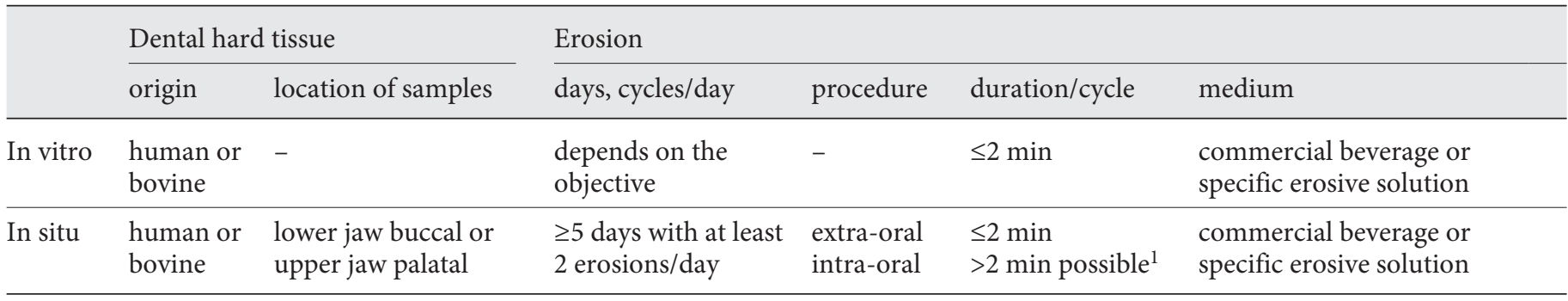

${ }^{1}$ If ethically justifiable. ${ }^{2}$ Standardisation recommended.

these models, the specimens are treated either in the oral cavity (in situ) or in the laboratory (in vitro). In some experiments, a combination of an in vitro and in situ approach has been used. For instance, samples are carried in the oral cavity in special appliances, but are demineralised and abraded by brushing under standardised in vitro conditions. The intention when using these set-ups is to apply standardised procedures which should simulate the intraoral conditions during demineralisation and abrasion as closely as possible. In other studies, the parameters are chosen to intentionally exaggerate clinical conditions. This is often done in order to create an amount of tissue loss which might be measurable with the chosen method. Other reasons for an exaggeration of the conditions are that a worst-case scenario should be tested, that the limits of protective methods should become discernible or that special parameters (e.g. impact of different brushing forces) should be examined. These exaggerated conditions are used for the above-mentioned reasons and will not be critically questioned in the following. Rather, the conditions chosen to mimic real everyday situations will be scrutinised in this critical review. Thus, the authors intend to discuss in how far the parameters chosen are able to simulate intra-oral clinical conditions in laboratory or in situ models as closely as possible.

\section{General Design}

A total of 62 in vitro and in situ studies dealing with abrasion of eroded dental hard tissue were extracted from the database PubMed using the following search terms: abrasion AND (acid OR erosion OR softening) AND (enamel OR dentine OR dentin) AND (in vitro OR in situ OR laboratory OR fluoride OR casein OR toothpaste OR dentifrice or toothbrushing OR brushing). Only full pa- pers in English were taken into consideration. Several parameters used in the experimental designs in these studies were tabulated. This voluminous material is available online as online supplementary tables S1 and S2 (for all online supplementary material, see www. karger.com/doi/10.1159/000325946). Our recommendations are summarised in table 1.

A total of 42 studies with an in vitro design could be identified (online suppl. table S1). Most of these studies used cyclic erosion/abrasion models rather than single brushing treatment of eroded dental hard tissues. The number of erosive/abrasive challenges showed a wide range from 3 [Betke et al., 2003; Wiegand et al., 2004; Vieira et al., 2006b] to 720 cycling treatments [Bartlett et al., 1994]. Remarkably, a trend to severe erosion (e.g. $>5 \mathrm{~min} /$ cycle) and/or extensive brushing (e.g. $>200$ brushing strokes/cycle) conditions could be observed in studies with very few cycles [Betke et al., 2003; Wiegand et al., 2004; Vieira et al., 2006b], while models using mild erosion and abrasion conditions were often performed at a higher frequency and repetition rate (e.g. 84 cycles consisting of $30 \mathrm{~s}$ of erosion and $15 \mathrm{~s}$ of abrasion, respectively [Lagerweij et al., 2006]). Some reasons for the exaggeration of the conditions have already been mentioned above, but it has to be pointed out that these extreme models are hardly able to simulate tooth wear adequately, particularly under in vitro conditions.

Twenty in situ studies were found in PubMed (online suppl. table S2). This means that the specimens were intraorally carried in individual appliances allowing their removal from the oral cavity for further treatments, such as demineralisation and brushing. Most of these studies applied cycling models with alternating de- and remineralisation phases. Alternating erosion/abrasion treatment also showed a great variety from 3 days with 2 cycles/ day [Hara et al., 2003; Turssi et al., 2004] up to 21 days with 


\begin{tabular}{|c|c|c|c|c|c|c|}
\hline \multirow{2}{*}{$\begin{array}{l}\text { Interim storage/intra-oral } \\
\text { exposure prior to brushing }\end{array}$} & \multicolumn{6}{|l|}{ Abrasion } \\
\hline & procedure & cycles & duration/cycle & toothbrush & toothpaste & brushing force \\
\hline $\begin{array}{l}\text { Recommended, storage in } \\
\text { artificial saliva }\end{array}$ & - & 2/day & $\begin{array}{l}\text { manual: } 10-15 \text { strokes } \\
\text { powered: } 5 \mathrm{~s}\end{array}$ & $\begin{array}{l}\text { powered or } \\
\text { manual }\end{array}$ & fluoride & $1-2 \mathrm{~N}^{2}$ \\
\hline $\begin{array}{l}\text { Recommended, intraoral } \\
\text { exposure }\end{array}$ & Intra- or extra-oral & 2/day & $\begin{array}{l}\text { manual: } 10-15 \text { strokes } \\
\text { powered: } 5 \mathrm{~s}\end{array}$ & $\begin{array}{l}\text { powered or } \\
\text { manual }\end{array}$ & fluoride & $1-2 \mathrm{~N}^{2}$ \\
\hline
\end{tabular}

3 cycles/day [Vieira et al., 2007]. Erosion and abrasion were mostly performed extra-orally, and only in a few studies was either demineralisation [Hooper et al., 2003] or brushing [Jaeggi and Lussi, 1999; Lussi et al., 2004] done with the appliances in situ. Although both intra-oral erosion and brushing of the samples are more likely to better simulate real conditions, it might be assumed that usually a higher standardisation could be achieved with samples eroded and brushed extra-orally under standardised conditions. Moreover, extra-oral handling minimises the risk of long-term acid exposure of the natural dentition. Statistical aspects of laboratory experiments (e.g. sample size) and in situ studies (e.g. subject and sample size, interand intra-individual salivary parameters) are not addressed in this review. However, to avoid random effects of the study conditions on a single day on a given volunteer, the cycles should run for a representative period of time, such as 5-7 successive days. If this is not applicable, the treatments should be repeated on different days to rule out random effects of a single-day condition.

Extra-oral administration of the erosive and abrasive challenges allows a high level of standardisation of the variables in both erosion (duration and $\mathrm{pH}$ ) and abrasion (duration and number of brushing strokes; kind of toothbrush and toothpaste, and brushing force). At the same time, however, this approach tends to produce a higher amount of wear than in the clinical situation, because modifying biological factors which are absent in vitro, such as dilution of the erosive solution by saliva or protection from erosion by the natural pellicle, cannot be simulated adequately. As a consequence, erosion and abrasion parameters, such as duration of erosion or amount of brushing strokes, must be carefully considered and probably diminished when performing erosion-abrasion research in laboratory experiments or in situ studies with the intention to simulate the clinical situation.

\section{Dental Hard Tissue}

Erosion/abrasion experiments use enamel and/or dentine samples of human (usually wisdom teeth) or bovine (incisors) origin. Under the same cyclic erosion-abrasion conditions, dentine mostly revealed a higher susceptibility to wear in both bovine and human teeth [Hooper et al., 2003; Turssi et al., 2004; Attin et al., 2007; Wegehaupt et al., 2008; Wiegand et al., 2008a; Ranjitkar et al., 2009; Wiegand et al., 2010]. As almost half of the studies used bovine rather than human samples, the question of whether bovine samples are an appropriate substitute for human teeth arises. In cyclic erosion/abrasion models, bovine enamel showed a slightly higher susceptibility to wear than human enamel both in vitro [Attin et al., 2007] and in situ [Rios et al., 2006b], while human and bovine dentine specimens did not perform differently under the same in vitro conditions [Wegehaupt et al., 2008]. Bovine samples have the advantage that up to $4-5$ specimens can mostly be gained out of a single bovine incisor. This allows for allocation of samples from the same tooth to different experimental groups, thus increasing comparability between those groups. Although it seems undoubtedly better to use human dental hard tissue to evaluate the impact of any kind of treatment, it seems to be acceptable to use bovine specimens instead of human ones, especially when relative tissue loss compared with controls or the relative effect of different agents is examined rather than absolute tissue loss.

In situ models require that the samples are intra-orally fixed in appliances in which they are usually placed either buccally in the lower jaw or palatally in the upper jaw. Both locations have advantages and disadvantages. In both locations, care has to be taken that no additional unintended abrasion of the sample surfaces caused by the buccal mucosa (lower jaw) or the tongue (upper jaw) in- 
terferes with the intended abrasion by tooth brushing. Additionally, at the palatal site, contact with saliva might be less intensive than in other regions of the oral cavity into which the major salivary glands empty. However, if the acid solution is applied by drinking, the palatal area is more exposed than the buccal area. Owing to these considerations, there seems to be no overall advantage of one of the two sites over the other, and either site could be chosen, taking the aim and set-up of the study into consideration.

\section{Erosion}

Duration of erosion varied between $15 \mathrm{~s}$ and $40 \mathrm{~min}$ (in vitro) and $40 \mathrm{~s}$ and $20 \mathrm{~min}$ (in situ) per cycle, while mostly an immersion time between 1 and $5 \mathrm{~min} /$ cycle was chosen. In only 1 single in situ study was erosion performed intra-orally by a 10-min exposure to acidic drinks 4 times per day for 10 days [Hooper et al., 2003]. The investigators checked the tissue loss intermittently to ensure that harm to the natural dentition was minimal and excluded participants in whom tissue loss exceeded a critical limit. Nevertheless, the design of the study led to 40 erosive challenges with a total of $400 \mathrm{~min}$ of exposure, which might be an ethical problem when using healthy volunteers for the experiment, since damage to the natural dentition could not be avoided completely.

Depending on the $\mathrm{pH}$ of the erosive agent, short erosion times of up to 3 min might result in a softened enamel layer of approximately $0.5 \mu \mathrm{m}$, which is prone to brushing wear [Wiegand et al., 2007b; Voronets and Lussi, 2010]. Considering that a high number of brushing strokes (approximately 500-1,000) was necessary to remove this softened enamel layer almost completely [Wiegand et al., 2007b; Voronets and Lussi, 2010], it does not seem reasonable to increase the immersion time above this value. Moreover, it should be noted that the intra-oral challenge to the natural dentition, for instance when drinking an acidic beverage, is less pronounced because of dilution by and interaction with saliva and because of uneven distribution of the solution in the oral cavity. This results in only a short period of about 2 min during which the $\mathrm{pH}$ at a tooth surface is below the critical $\mathrm{pH}$ [Millward et al., 1997]. Thus, when performing the erosion extra-orally, exposure to the acidic environment without salivary interaction should not exceed a period of $2 \mathrm{~min} /$ cycle.

Less information regarding the abrasion-prone layer of erosion-affected dentine is currently available. Longtime erosion for $2 \mathrm{~h}$ led to a surface softening of $2-4 \mu \mathrm{m}$, which could be removed by ultrasonication [Vanuspong et al., 2002]. It can only be speculated how much of this softened dentine surface could be removed by brushing and whether shorter erosion times would produce a distinctly smaller softened zone. However, to allow comparison between enamel and dentine samples and to ensure a realistic duration, short erosion periods should be preferred to long ones.

Regarding the erosion medium, many studies used commercial beverages, while others used citric or hydrochloric acid at a specific $\mathrm{pH}$. Although it makes sense to use soft drinks in order to simulate the everyday situation, it seems also appropriate to use specific acidic solutions to test special properties and parameters of erosive media.

\section{Interim Storage (in vitro) and Intra-Oral Exposure (in situ) prior to Brushing}

In almost half of the studies, the samples were not brushed immediately after erosion, but with a time delay of up to $4 \mathrm{~h}$ (mostly 30-60 min) to increase the abrasion resistance of eroded enamel and dentine. The efficacy of waiting periods to increase abrasion resistance is still questioned, since data are conflicting. Some studies show that abrasion resistance is significantly increased by storage in artificial saliva [Attin et al., 2000] or by intra-oral exposure [Jaeggi and Lussi, 1999; Attin et al., 2001b, 2004; Rios et al., 2006b], while others found no effect [Attin et al., 2001a; Hara et al., 2003; Ganss et al., 2007b; SalesPeres et al., 2007; Kato et al., 2009]. However, a time delay before brushing might better represent conditions in daily life, as it seems rather unlikely that people brush their teeth immediately after each erosive challenge.

In laboratory experiments, samples were usually stored in artificial saliva for 1-60 min, while only 3 studies used human saliva as the storage medium [Kelly and Smith, 1988; Attin et al., 2001a; Hara et al., 2008]. Currently, it is unclear whether human or artificial saliva is more suitable as the immersion medium in vitro, as this was only examined in 1 study so far [Hara et al., 2008]. Although current in vitro models use a wide range of different saliva substitutes, artificial saliva provides the advantage that it can be prepared in sufficient amounts and with a consistent composition, which leads to a high degree of standardisation. Human saliva can be either collected from one donor [Kelly and Smith, 1988] or collected from several donors and pooled [Attin et al., 2001a; Hara et al., 2008]. Besides the problems that the composition of human saliva might show a high intra- and inter-sample variability and that 
large volumes of saliva are usually necessary in laboratory models, components of human saliva might be rapidly altered or degraded under in vitro conditions. Hence, it seems questionable whether the use of human saliva provides a crucial benefit in an in vitro set-up compared to saliva substitutes and hence whether the use of human saliva mirrors the clinical situation more closely. Thus, based on the current knowledge, the use of artificial saliva as an immersion medium prior to brushing seems to be appropriate if a delayed abrasion treatment is planned.

Generally, to simulate the everyday situation, brushing of eroded dental hard tissues should be delayed by storage in artificial saliva in vitro or by intra-oral exposure in situ. To omit intra-oral exposure to saliva before brushing only makes sense when an experiment is designed to evaluate immediate brushing of eroded surfaces.

\section{Brushing Abrasion}

Online supplementary tables S1 and S2 evidence that in abrasion experiments, there is considerable variation in several brushing parameters, namely the frequency and duration of brushing, the kind of toothbrush and toothpaste used, and the brushing force applied. Most cyclic models used an alternating treatment with equal numbers of erosive and abrasive challenges, which means that each erosive challenge is followed by brushing, irrespective of the waiting period chosen. Only a few in vitro studies used a different set-up in which the samples were subjected to 6 erosive challenges but only 2 brushing cycles/day [Lagerweij et al., 2006; Ganss et al., 2007b, 2009a]. In addition, 3 in situ studies modified the alternating treatments in such a way that fewer abrasive than erosive challenges were performed each day [Vieira et al., 2007; Magalhaes et al., 2009; Wiegand et al., 2010]. This approach might reflect the clinical situation better, as most people brush their teeth twice daily [Ganss et al., 2009b] rather than after each contact with erosive food or beverages.

In the majority of studies, the samples were brushed with a manual toothbrush (25-5,000 strokes/cycle), while fewer studies used electric toothbrushes ( $5 \mathrm{~s}$ to $1 \mathrm{~min}$ per cycle). Generally, laboratory experiments used a more severe abrasion treatment (duration or number of brushing strokes) than in situ studies. However, clinical surveys show that the overall brushing time for the whole dentition amounts to $30-90 \mathrm{~s}$, which is equivalent to $300-$ 400 brushing strokes [Macgregor and Rugg-Gunn, 1979, 1985; Ganss et al., 2009b]. Thus, it becomes evident that the regimes applied in most in vitro and in situ models exceed clinical conditions distinctly. Considering that, in vivo, each tooth might receive 10-15 brushing strokes with a manual toothbrush or less than $5 \mathrm{~s}$ of brushing with an electrical toothbrush under clinical conditions, only $20 \%$ of the in vitro studies [Ponduri et al., 2005; Hemingway et al., 2006; Ganss et al., 2007b; Wiegand et al., 2008b; Ganss et al., 2009a; Wiegand et al., 2009; Moretto et al., 2010] and 60\% of the in situ studies almost fulfilled this condition [Attin et al., 2001b; Hara et al., 2003; Attin et al. 2004; Turssi et al., 2004; Rios et al., 2006; Sales-Peres et al., 2007; Vieira et al., 2007; Rios et al., 2008a; Wiegand et al., 2008a; Magalhaes et al., 2009].

In vitro, usually both manual and electrical toothbrushes are fixed in automatic brushing machines or toothbrush holders which ensure a standardised movement of the brush over the sample surface as well as a constant brushing force [Parry et al., 2008]. Brushing forces vary between 0.2 and $4.5 \mathrm{~N}$, but most of the studies used a brushing force of $2-3 \mathrm{~N}$. These values match the clinical values of the mean load applied during brushing with a manual toothbrush [Ganss et al., 2009b]. It might be reasonable to reduce the brushing force of powered brushes to 1.5 or $2 \mathrm{~N}$ [Vieira et al., 2006; Ganss et al., 2007b], not least since powered toothbrushes have shown a higher potential to damage eroded dental hard tissues than manual brushes at the same force [Wiegand et al., 2006].

In most in situ studies, brushing was conducted extraorally, with the exception of two studies [Jaeggi and Lussi, 1999; Lussi et al., 2004] in which the samples were brushed intra-orally, while the appliances with the specimens were still in the oral cavity. In all studies, a high variability of tissue loss was observed, irrespective of whether samples were brushed intra- or extra-orally. This might be partly explained by differences in brushing forces used by the volunteers, even though they might be carefully trained and instructed. One possibility to achieve a higher level of standardisation might be the use of brushing machines or holders [Wiegand et al., 2008a, 2010], the adjustment of a constant brushing force by scales [Ganss et al., 2007b] or brushing by the same person (investigator) [Hooper et al., 2003; Vieira et al., 2007]. Thus, there seems to be no distinct benefit in performing intra-oral brushing of the samples fixed in the appliances.

Various manual and powered toothbrushes are used in vitro. However, although the abrasion potential of toothbrushes is influenced by the brushing force [Wiegand et al., 2007a; Ganss et al., 2009a], the type of brush and the filament stiffness [Wiegand et al., 2008b, 2009], the impact of the toothbrush per se is considered to be significantly lower than the impact of the toothpaste [Wiegand et al., 
2008b, 2009; Hara et al., 2009]. The abrasivity and the fluoride content are considered relevant toothpaste parameters in erosion/abrasion experiments. Unfortunately, most of the published studies do not refer to the abrasivity [REA (enamel abrasion) or RDA (dentine abrasion) value] of the toothpaste, even though several studies indicate that abrasion of eroded enamel and dentine depends strongly on this factor [Hooper et al., 2003; Wiegand et al., 2008b; Hughes et al., 2008; Hara et al., 2009; Wiegand et al., 2009]. In contrast, authors usually specified whether they were using fluoridated or non-fluoridated toothpastes. Fluoridated toothpastes produced less wear on eroded enamel and dentine than non-fluoridated toothpastes both in vitro [Bartlett et al., 1994; Lagerweij et al., 2006; Hara et al., 2009] and in situ [Ganss et al., 2007b; Magalhaes et al., 2007, 2008]. Depending on the objective of the study, the use of either fluoridated or non-fluoridated toothpaste might be suitable, but it has to be considered that most toothpastes available on the international market contain fluoride. Thus, whenever possible, fluoride-containing toothpastes should be used to simulate clinical conditions.

\section{Conclusion and Recommendations}

This review aimed to characterise and critically discuss the different designs of published in vitro and in situ models and to describe some important factors which influence the outcome of the respective studies and thus need to be considered carefully. Based on this review, the authors recommend variables to be considered in ero- sion/abrasion studies (table 1) aiming to simulate the intra-oral everyday situation of patients suffering from erosion as closely as possible. These recommendations should be understood as guidelines rather than as a standard schedule, although it has to be considered that a higher level of standardisation among experiments would allow better comparison of study outcomes.

In vitro studies are important to clarify and estimate the relative effects of brushing of eroded dental hard tissue and the role of new anti-erosive methods or compounds, although in situ models have the potential to study fundamental aspects of the development of the erosion/abrasion processes as closely as possible to the clinical situation without affecting the natural dentition.

The challenge of both laboratory and in situ studies is to strike a balance between the intention to simulate clinical conditions and the need to conduct experiments in a rational and practicable way as well as in a short time span and to gain measurable results. The variables suggested in table 1 are applied in a very short experiment (either in situ or in vitro), so the amount of tissue loss might be below the detection limit of the assessment techniques, such as profilometry or microradiography, which are usually employed in erosion research [Attin, 2006]. This conversely emphasises the need for further development in this field.

\section{Disclosure Statement}

The authors declare that there are no potential conflicts of interest.

\section{References}

Attin T: Methods for assessment of dental ero- Attin T, Siegel S, Buchalla W, Lennon AM, Hansion; in Lussi A: Dental Erosion. From Diagnosis to Therapy. Monogr Oral Sci. Basel, Karger, 2006, vol 20, pp 152-172.

Attin T, Buchalla W, Gollner M, Hellwig E: Use of variable remineralization periods to improve the abrasion resistance of previously eroded enamel. Caries Res 2000;34:48-52.

Attin T, Buchalla W, Putz B: In vitro evaluation of different remineralization periods in improving the resistance of previously eroded bovine dentine against tooth-brushing abrasion. Arch Oral Biol 2001a;46:871-874.

Attin T, Knofel S, Buchalla W, Tutuncu R: In situ evaluation of different remineralization periods to decrease brushing abrasion of de-
mineralized enamel. Caries Res 2001b;35: 216-222. nig $\mathrm{C}$, Becker K: Brushing abrasion of softened and remineralised dentin: an in situ study. Caries Res 2004;38:62-66.

Attin T, Wegehaupt F, Gries D, Wiegand A: The potential of deciduous and permanent bovine enamel as substitute for deciduous and permanent human enamel: erosion-abrasion experiments. J Dent 2007;35:773-777.

Bartlett DW, Smith BG, Wilson RF: Comparison of the effect of fluoride and non-fluoride toothpaste on tooth wear in vitro and the influence of enamel fluoride concentration and hardness of enamel. Br Dent J 1994;176: 346-348.

Betke H, Schick U, Buchalla W, Hellwig E, Attin $\mathrm{T}$ : Influence of the buffer capacity of amine fluoride-containing toothpastes and gels in enamel erosion. Schweiz Monatsschr Zahnmed 2003;113:1158-1164.
Ganss C, Hardt M, Blazek D, Klimek J, Schlueter $\mathrm{N}$ : Effects of toothbrushing force on the mineral content and demineralized organic matrix of eroded dentine. Eur J Oral Sci 2009a; 117:255-260.

- Ganss C, Schlueter N, Hardt M, von Hinckeldey J, Klimek J: Effects of toothbrushing on eroded dentine. Eur J Oral Sci 2007b;115:390396.

Ganss C, Schlueter N, Preiss S, Klimek J: Tooth brushing habits in uninstructed adults - frequency, technique, duration and force. Clin Oral Investig 2009b;13:203-208.

Hara AT, Gonzalez-Cabezas C, Creeth J, Parmar M, Eckert GJ, Zero DT: Interplay between fluoride and abrasivity of dentifrices on dental erosion-abrasion. J Dent 2009;37:781785 . 
- Hara AT, Gonzalez-Cabezas C, Creeth J, Zero DT: The effect of human saliva substitutes in an erosion-abrasion cycling model. Eur J Oral Sci 2008;116:552-556.

- Hara AT, Turssi CP, Teixeira EC, Serra MC, Cury JA: Abrasive wear on eroded root dentine after different periods of exposure to saliva in situ. Eur J Oral Sci 2003;111:423-427.

-Hemingway CA, Parker DM, Addy M, Barbour ME: Erosion of enamel by non-carbonated soft drinks with and without toothbrushing abrasion. Br Dent J 2006;201:447-450.

-Hooper S, West NX, Pickles MJ, Joiner A, Newcombe RG, Addy M: Investigation of erosion and abrasion on enamel and dentine: a model in situ using toothpastes of different abrasivity. J Clin Periodontol 2003;30:802-808.

-Hughes N, Mason S, Creeth J, Hara AT, Parmar M, Gonzalez-Cabezas C: The effect of antisensitivity dentifrices on brushing abrasion of eroded dentin in vitro. J Clin Dent 2008; 19:143-146.

Jaeggi T, Lussi A: Toothbrush abrasion of erosively altered enamel after intraoral exposure to saliva: an in situ study. Caries Res 1999;33:455-461.

-Kato MT, Magalhaes AC, Rios D, Hannas AR, Attin T, Buzalaf MA: Protective effect of green tea on dentin erosion and abrasion. J Appl Oral Sci 2009; 17:560-564.

Kelly MP, Smith BG: The effect of remineralizing solutions on tooth wear in vitro. J Dent 1988; 16:147-149.

- Lagerweij MD, Buchalla W, Kohnke S, Becker K, Lennon AM, Attin T: Prevention of erosion and abrasion by a high fluoride concentration gel applied at high frequencies. Caries Res 2006;40:148-153.

Lussi A, Jaeggi T, Gerber C, Megert B: Effect of amine/sodium fluoride rinsing on toothbrush abrasion of softened enamel in situ. Caries Res 2004;38:567-571.

Macgregor ID, Rugg-Gunn AJ: Survey of toothbrushing duration in 85 uninstructed English schoolchildren. Community Dent Oral Epidemiol 1979;7:297-298.

Macgregor ID, Rugg-Gunn AJ: Toothbrushing duration in 60 uninstructed young adults. Community Dent Oral Epidemiol 1985;13: 121-122.

-Magalhaes AC, Rios D, Delbem AC, Buzalaf MA, Machado MA: Influence of fluoride dentifrice on brushing abrasion of eroded human enamel: an in situ/ex vivo study. Caries Res 2007:41:77-79.

-Magalhaes AC, Rios D, Moino AL, Wiegand A, Attin T, Buzalaf MA: Effect of different concentrations of fluoride in dentifrices on dentin erosion subjected or not to abrasion in situ/ex vivo. Caries Res 2008;42:112-116.
Magalhaes AC, Wiegand A, Rios D, Hannas A, Attin T, Buzalaf MA: Chlorhexidine and green tea extract reduce dentin erosion and abrasion in situ. J Dent 2009;37:994-998.

-Millward A, Shaw L, Harrington E, Smith AJ: Continuous monitoring of salivary flow rate and $\mathrm{pH}$ at the surface of the dentition following consumption of acidic beverages. Caries Res 1997;31:44-49.

Moretto MJ, Magalhaes AC, Sassaki KT, Delbem AC, Martinhon CC: Effect of different fluoride concentrations of experimental dentifrices on enamel erosion and abrasion. Caries Res 2010;44:135-140.

Parry J, Harrington E, Rees GD, McNab R, Smith AJ: Control of brushing variables for the in vitro assessment of toothpaste abrasivity using a novel laboratory model. J Dent 2008;36: 117-124.

Ponduri S, Macdonald E, Addy M: A study in vitro of the combined effects of soft drinks and tooth brushing with fluoride toothpaste on the wear of dentine. Int J Dent Hyg 2005; 3:7-12.

Ranjitkar S, Rodriguez JM, Kaidonis JA, Richards LC, Townsend GC, Bartlett DW: The effect of casein phosphopeptide-amorphous calcium phosphate on erosive enamel and dentine wear by toothbrush abrasion. J Dent 2009;37:250-254.

- Rios D, Honorio HM, Magalhaes AC, Buzalaf MA, Palma-Dibb RG, Machado MA, da Silva SM: Influence of toothbrushing on enamel softening and abrasive wear of eroded bovine enamel: an in situ study. Braz Oral Res 2006a;20:148-154.

-Rios D, Honorio HM, Magalhaes AC, Delbem AC, Machado MA, Silva SM, Buzalaf MA: Effect of salivary stimulation on erosion of human and bovine enamel subjected or not to subsequent abrasion: an in situ/ex vivo study. Caries Res 2006b;40:218-223.

Rios D, Honorio HM, Magalhaes AC, Silva SM, Delbem AC, Machado MA, Buzalaf MA: Scanning electron microscopic study of the in situ effect of salivary stimulation on erosion and abrasion in human and bovine enamel. Braz Oral Res 2008a;22:132-138.

- Sales-Peres SH, Pessan JP, Buzalaf MA: Effect of an iron mouthrinse on enamel and dentine erosion subjected or not to abrasion: an in situ/ex vivo study. Arch Oral Biol 2007;52: $128-132$.

- Turssi CP, Faraoni JJ, Rodrigues AL Jr, Serra MC: An in situ investigation into the abrasion of eroded dental hard tissues by a whitening dentifrice. Caries Res 2004;38:473-477.

Vanuspong W, Eisenburger M, Addy M: Cervical tooth wear and sensitivity: erosion, softening and rehardening of dentine; effects of $\mathrm{pH}$, time and ultrasonication. J Clin Periodontol 2002;29:351-357.
Vieira A, Jager DH, Ruben JL, Huysmans MC: Inhibition of erosive wear by fluoride varnish. Caries Res 2007;41:61-67.

-Vieira A, Lugtenborg M, Ruben JL, Huysmans MC: Brushing abrasion of eroded bovine enamel pretreated with topical fluorides. Caries Res 2006a;40:224-230.

-Vieira A, Overweg E, Ruben JL, Huysmans MC: Toothbrush abrasion, simulated tongue friction and attrition of eroded bovine enamel in vitro. J Dent 2006b;34:336-342.

Voronets J, Lussi A: Thickness of softened human enamel removed by toothbrush abrasion: an in vitro study. Clin Oral Investig 2010;14:251-256.

Wegehaupt F, Gries D, Wiegand A, Attin T: Is bovine dentine an appropriate substitute for human dentine in erosion/abrasion tests? J Oral Rehabil 2008;35:390-394.

Wiegand A, Begic M, Attin T: In vitro evaluation of abrasion of eroded enamel by different manual, power and sonic toothbrushes. Caries Res 2006a;40:60-65.

Wiegand A, Egert S, Attin T: Toothbrushing before or after an acidic challenge to minimize tooth wear? An in situ/ex vivo study. Am J Dent 2008a;21:13-16.

-Wiegand A, Hiestand B, Sener B, Magalhaes AC, Roos M, Attin T: Effect of $\mathrm{TiF}_{4}, \mathrm{ZrF}_{4}, \mathrm{HfF}_{4}$ and $\mathrm{AmF}$ on erosion and erosion/abrasion of enamel and dentin in situ. Arch Oral Biol 2010;55:223-228.

-Wiegand A, Kowing L, Attin T: Impact of brushing force on abrasion of acid-softened and sound enamel. Arch Oral Biol 2007a;52: 1043-1047.

Wiegand A, Kuhn M, Sener B, Roos M, Attin T: Abrasion of eroded dentin caused by toothpaste slurries of different abrasivity and toothbrushes of different filament diameter. J Dent 2009;37:480-484.

-Wiegand A, Lemmrich F, Attin T: Influence of rotating-oscillating, sonic and ultrasonic action of power toothbrushes on abrasion of sound and eroded dentine. J Periodontal Res 2006b;41:221-227.

Wiegand A, Schwerzmann M, Sener B, Magalhaes AC, Roos M, Ziebolz D, Imfeld T, Attin $\mathrm{T}$ : Impact of toothpaste slurry abrasivity and toothbrush filament stiffness on abrasion of eroded enamel - an in vitro study. Acta Odontol Scand 2008b;66:231-235.

-Wiegand A, Wegehaupt F, Werner C, Attin T: Susceptibility of acid-softened enamel to mechanical wear - ultrasonication versus toothbrushing abrasion. Caries Res 2007b; 41:56-60.

Wiegand A, Wolmershauser S, Hellwig E, Attin $\mathrm{T}$ : Influence of buffering effects of dentifrices and fluoride gels on abrasion on eroded dentine. Arch Oral Biol 2004;49:259-265. 